\author{
ACTA AGROBOTANICA \\ Vol. 64 (2): 93-108 \\ 2011
}

\title{
SEGETAL FLORA OF THE ŁUKÓW PLAIN
}

\section{Zofia Rzymowska, Teresa Skrajna}

\author{
The Agricultural Ecology Department of the Siedlce University of Natural Sciences and Humanities, \\ B. Prusa 14, 08-110 Siedlce, Poland, \\ e-mail: ekorol@uph.edu.pl
}

Received: 20.07.2010

\begin{abstract}
The aim of the study was to characterize and analyze the segetal flora of the Łuków Plain. The study was carried out from 2003 to 2006 in 182 towns and villages. Vascular flora of the arable fields in the area under study consists of 305 species belonging to 39 families and 168 genera. The analysed flora is characterised by the prevalence of native species (64.6\%) over alien species (35.4\%). Archaeophytes dominate among anthropophytes, whereas meadow species are the most frequent in the group of apophytes. Annuals and biennials show a slight prevalence over perennials. The analysis of the life-form categories shows the dominance of therophytes $(52.8 \%)$ as well as a relatively high share of hemicryptophytes $(34.4 \%)$ and geophytes $(12.5 \%)$. In the analysed flora, rare and very rare species constitute a vast majority $(61.7 \%)$, whereas the common and very common species reach $13.1 \%$.
\end{abstract}

Key words: archeophytes, apophytes, kenophytes, Sub-Atlantic species, speirochoric species

\section{INTRODUCTION}

Segetal flora of the Łuków Plain so far has not been the object of a complete study. Data on the occurrence of vascular plants in the area can be found in the papers by Ci ose k and S krzy c zy ńska (1989), Ciosek et al. (1997), Ciosek and Pikulski (1997), but these data mainly concern other kinds of vegetation. The geographical ranges of some SubAtlantic species, e.g. Arnoseris minima and Teesdalea nudicaulis, run across this region. They are considered rare and endangered plants in many regions of Poland and in Europe (Fijałk ow ski and Nycz, 1998; Urbisz et al. 1998; Siciński, 1998; Kubat and Kaplan, 2001; Prasse et al. 2001; Cheffings and Farre11, 2005; Pinke et al. 2006).
The reason for their regression is an increase in the area of fallow lands, afforestation of poor soils and their high sensitivity to herbicides, and high fertilization level (Fijałk ow ski, 1994; Fi jałk ow ski and Ny c z, 1998; S i c ińs ki, 1998). The Łuków Plain is a typically agricultural mezoregion of rather traditional farming methods. For this reason, the numerous occurrence of speirochoric species in segetal flora of the studied area was noted.

These features of the area as well as the lack of studies on the flora of arable fields inspired the author to undertake this study on the Luków Plain area. The aim of the study was to investigate and analyse the flora of the Łuków Plain, with a special attention to speirochoric and Sub-Atlantic species.

\section{THE AREA UNDER STUDY}

The Łuków Plain covers an area of about 2600 $\mathrm{km}^{2}$ and stretches from Adamów and Kock to the Bug River Valley between Terespol and Mielnik (Kon$\mathrm{d} \mathrm{r} \mathrm{a} \mathrm{c} \mathrm{k} \mathrm{i,} \mathrm{2002)} \mathrm{over} \mathrm{the} \mathrm{territories} \mathrm{of} \mathrm{several} \mathrm{districts:}$ Łuków, Siedlce, Łosice, Radzyń, and Biała Podlaska Districts. According to the physiographic division, the area belongs to the macroregion of the South Podlachian Lowland, which is a part of the province of the Central Polish Lowlands. It is the furthest to the east area of the Central European Lowland. The land features are not significantly diversified. The areas of denudation plains, sometimes slightly undulating, prevail here. The sediments of the Central Polish glaciation, mainly of the Odra Stage, primarily clays and sands of the basal moraine, were mixed and diluted during periglacial processes related to transgression and regression of the glacier. Fluvioglacial sands lying on different sediment levels in some areas are visible on the 
surface in the form of sands and clays. Sand forms are usually mixed with dust fraction. In the river valleys and land hollows, contemporary sediments developed in the form of peat on the mineral base and boggy soil. Poor soils prevail in the studied area (class IVb $-29 \%$ and V - 29\%), whereas the best soils are extremely rare. From the floristic point of view, the Łuków Plain is an area of great interest, which mainly results from the fact that the north-eastern range boundaries of many species, for instance Abies alba and Daphne cneorum, run through this area.

\section{METHODS}

The field studies were carried out from 2003 to 2006 in 182 towns and villages in 19 communes. The studied region is shown in Fig. 1. Lists of plant species and 815 phytosociological relevés made according to the Braun-Blanquet method in cereal and root crops as well as in stubble fields constituted the material for flora analysis. The studies were carried out in cultivated fields, excluding balks, roadsides, and fallow lands. Taxonomic differences, the biological spectrum and geohistorical structure of the flora, along with stability and frequency of occurrence of the species, are presented in the paper. The used taxonomy is according to R u tk ow ski (1998) and species nomenclature follows Mirek et al. (2002). In defining the features of the species, the following papers were used: Aniol-Kwiatkowska (1974), Kornaś (1968, 1977), Korniak (1992), Rutkowski (2007), Sowa and Warcholińska (1987), Zając (1979), Zając and Zając (1975, 1992), $\mathrm{Z}$ a j ą c et al. (1998). The frequency of occurrence of the taxa is given according to the pre-arranged formula stating that the number of stands is equal to the number of towns and villages, where the following categories: very rare, rare, quite rare, frequent, quite common and very common, occur in 1-3, 4-14, 15-36, 37-72, 73-109, 110-145 and 146-182 stands, respectively.

\section{RESULTS}

The segetal flora of the Łuków Plain consists of 305 species belonging to 39 botanical families and 168 genera. Over $70 \%$ of the taxa are in the ten families most abundant in species. The most numerously represented families are as follows: Asteraceae (51 species and 34 genera), Poaceae (33 species and 20 genera), Fabaceae (24 species and 6 genera), and Brassicaceae (22 species and 18 genera) (Table 1 ).

A characteristic feature of the flora under discussion is the considerable prevalence of apophytes over anthropophytes: 197 species $(64.6 \%)$ and 108 species (35.4\%), respectively (Fig. 2). A clear dominance of species from meadow habitats can be observed among apophytes: 72 species (36.5\%). Waterside and marshy habitat taxa (40 species $-20.3 \%$ ), apophytes of dunes and sands (34 species - 17.3\%) and forest taxa (29 species $-14.7 \%$ ) have a lesser contribution, and thermophilous grasslands with 22 species $(11.2 \%)$ have the smallest contribution (Fig. 3).

Archaeophytes prevail among alien species (83 species $-27.3 \%$ ). Special attention is drawn to the fact that speirochoric species, such as Agrostemma githago, Bromus secalinus and Avena strigosa, are frequently found there. Moreover, this group also consists of common and resilient field weeds, such as: Apera spica-venti, Echinochloa crus-galli, Fallopia convolvulus, Raphanus raphanistrum, Capsella bursa-pastoris, Anthemis arvensis, and Matricaria maritima subsp. inodora. Epecophytes are represented by 19 species $(6.2 \%)$ and ergasiophytes by 6 species (2.0\%) (Fig. 1). Common kenophytes of the area under study are Galinsoga parviflora and Conyza canadensis. The mass occurrence and invasive nature of species such as Vicia grandiflora, Bunias orientalis and Anthoxanthum aristatum in some localities are also especially noteworthy.

Therophytes with 161 species (52.8\%), particularly among anthropophytes (92 species $-85.2 \%$ ) (Fig. 4), are the dominant life-form of the flora in question. Hemicryptophytes are quite numerously represented by 105 species (34.5\%), particularly among apophytes (94 species $-47.7 \%$ ). There are 38 species of geophytes (12.5\%) and one chamephyte.

Analysing the stability of the flora of the Euków Plain, the prevalence of annuals and biennials (173 species $-56.7 \%$ ) over perennials (132 species $-43.3 \%$ ) can be observed. The short lifespan is characteristic for anthropophytes (98 species $-90.7 \%$ ), whereas among apophytes perennials prevail (122 species $-61.9 \%$ ) (Fig. 5).

In the analysed flora, rare and very rare species are dominant, constituting $61.6 \%$ of the flora. There are 40 common and very common species (13.1\%). 
Table 1.

Systematic list of species

\begin{tabular}{|c|c|c|c|c|}
\hline Family and species name & $\begin{array}{l}\text { Geographic- } \\
\text { historical } \\
\text { group }\end{array}$ & Persistence & Life form & Frequency \\
\hline \multicolumn{5}{|l|}{ Equisetaceae } \\
\hline 1. Equisetum arvense L. & Am & $\mathrm{P}$ & $\mathrm{G}$ & very common \\
\hline 2. E. sylvaticum $\mathrm{L}$. & $\mathrm{Am}$ & $\mathrm{P}$ & G & rare \\
\hline 3. E. palustre L. & $\mathrm{Am}$ & $\mathrm{P}$ & G & very rare \\
\hline \multicolumn{5}{|l|}{ Urticaceae } \\
\hline 4. Urtica urens L. & Ar & A & $\mathrm{T}$ & rare \\
\hline 5. U. dioica $\mathrm{L}$. & Af & $\mathrm{P}$ & $\mathrm{G}(\mathrm{H})$ & very rare \\
\hline \multicolumn{5}{|l|}{ Polygonaceae } \\
\hline 6. Polygonum aviculare L. & Aw & A & $\mathrm{T}$ & common \\
\hline 7. P. bistorta L. & Am & $\mathrm{P}$ & G & very rare \\
\hline 8. P. amphibium $\mathrm{L}$. & Aw & $\mathrm{P}$ & $\mathrm{G}$ & frequent \\
\hline 9. P. hydropiper L. & Aw & A & $\mathrm{T}$ & rather common \\
\hline 10. P. mite Schrank & Aw & A & $\mathrm{T}$ & very rare \\
\hline 11. P. minus Huds. & Aw & A & $\mathrm{T}$ & rather rare \\
\hline 12. P. persicaria $\mathrm{L}$. & Aw & A & $\mathrm{T}$ & rather common \\
\hline 13. P. lapathifolium L. subsp. pallidum (With.) Fr. & Aw & A & $\mathrm{T}$ & common \\
\hline 14. P. lapathifolium L. subsp. lapathifolium & Aw & A & $\mathrm{T}$ & common \\
\hline 15. Fallopia convolvulus (L.) A. Löve & $\mathrm{Ar}$ & A & $\mathrm{T}$ & very common \\
\hline 16. Rumex acetosella $\mathrm{L}$. & As & $\mathrm{P}$ & $\mathrm{G}(\mathrm{H})$ & very common \\
\hline 17. R. acetosa L. & Am & $\mathrm{P}$ & $\mathrm{H}$ & very rare \\
\hline 18. R. obtusifolius L. & Af & $\mathrm{P}$ & $\mathrm{G}$ & rather rare \\
\hline 19. R. crispus L. & $\operatorname{Am}(w)$ & $\mathrm{P}$ & G & frequent \\
\hline \multicolumn{5}{|l|}{ Chenopodiaceae } \\
\hline 20. Chenopodium glaucum L. & Aw & A & $\mathrm{T}$ & very rare \\
\hline 21. Ch. hybridum L. & Ar & A & $\mathrm{T}$ & rare \\
\hline 22. Ch. polyspermum L. & Aw & A & $\mathrm{T}$ & rare \\
\hline 23. Ch. album L. & Aw & A & $\mathrm{T}$ & very common \\
\hline 24. Atriplex patula L. & $\mathrm{Ar}$ & A & $\mathrm{T}$ & rare \\
\hline \multicolumn{5}{|l|}{ Amaranthaceae } \\
\hline 25. Amaranthus retroflexus L. & Ep & A & $\mathrm{T}$ & rather rare \\
\hline 26. A. chlorostachys Willd. & Ep & A & $\mathrm{T}$ & very rare \\
\hline 27. A. lividus L. & Ep & A & $\mathrm{T}$ & very rare \\
\hline \multicolumn{5}{|l|}{ Caryophyllaceae } \\
\hline 28. Arenaria serpyllifolia L. & At & A & $\mathrm{T}$ & rather rare \\
\hline 29. Stellaria media (L.) Vill. & $\operatorname{Am}(f)$ & A & $\mathrm{T}$ & very common \\
\hline 30. S. graminea L. & Am & $\mathrm{P}$ & $\mathrm{H}$ & rather rare \\
\hline 31. Holosteum umbellatum L. & As & A & $\mathrm{T}$ & very rare \\
\hline 32. Cerastium arvense L. & As & $\mathrm{P}$ & $\mathrm{H}(\mathrm{Ch})$ & rare \\
\hline 33. C. holosteoides Fr. em. Hyl. & Am & A & $\mathrm{H}$ & common \\
\hline 34. C. semidecandrum L. & At & A & $\mathrm{T}$ & rather rare \\
\hline
\end{tabular}




\section{Sagina procumbens L.}

36. Scleranthus annuus L.

37. Herniaria glabra L.

38. Spergula arvensis $\mathrm{L}$.

39. S. arvensis L. subsp. maxima (Weihe) O.Schwarz

40. S. morisonii Boreau

41. Spergularia rubra (L.) J. Presl et C. Persl .

42. Agrostemma githago L.

43. Melandrium album (Mill.) Garcke

44. M. noctiflorum L.

45. Gypsophila muralis L.

46. Saponaria officinalis L.

Ranunculaceae

47. Consolida regalis S.F. Gray

48. Ranunculus flammula $\mathrm{L}$.

49. R. repens L.

50. R. sardous Crantz

51. R. acris L. S.str.

52. Myosurus minimus L.

Papaveraceae

53. Papaver somniferum L.

54. P. argemone $\mathrm{L}$.

55. P. dubium L.

56. P. rhoeas L.

57. Chelidonium majus L.

Fumariaceae

58. Fumaria officinalis L.

Brassicaceae

59. Sisymbrium officinale (L.) Scop.

60. S. altissimum L.

61. Descurainia sophia (L.) Webb. ex Prantl

62. Arabidopsis thaliana (L.) Heynh.

63. Bunias orientalis L.

64. Erysimum cheiranthoides L.

65. Rorippa palustris (L.) Besser

66. R. sylvestris (L.) Besser

67. Armoracia rusticana P. Gaertn., B. Mey. et Scherb.

68. Cardaminopsis arenosa (L.) Hayek

69. Berteroa incana (L.) DC

70. Erophila verna (L.) Chevall.

71. Neslia paniculata (L.) Desv.

72. Capsella bursa-pastoris (L.) Medik.

73. Teesdalea nudicaulis (L.) R. Br.

74. Thlaspi arvense L.

$\mathrm{Am}(\mathrm{w})$
$\mathrm{Ar}$
$\mathrm{As}$
$\mathrm{Ar}$
$\mathrm{Ar}$
$\mathrm{As}$
$\mathrm{Aw}$
$\mathrm{Ar}$
$\mathrm{Am}$
$\mathrm{Ar}$
$\mathrm{Aw}$
$\mathrm{Aw}$

$\mathrm{P}$

A

A

A

A

A

A

A

A

A

A

$\mathrm{P}$

Ar

A $T$

$\mathrm{T}$

Aw

$\mathrm{Am}(\mathrm{w})$

$\mathrm{P}$

$\mathrm{H}$

$\mathrm{H}$

Am $P$

Am

P

Aw

A

$\mathrm{H}$

$\mathrm{T}$

$\mathrm{H}$

rather rare

common

very rare

common

very rare

very rare

frequent

rather rare

frequent

rather rare

rather common

very rare

(H)

rather rare

very rare

frequent

rather rare

very rare

rare

Er
Ar
Ar
Ar
Af

$\mathrm{A}$
$\mathrm{A}$
$\mathrm{A}$
$\mathrm{A}$
$\mathrm{P}$

$\mathrm{T}$
$\mathrm{T}$
$\mathrm{T}$
$\mathrm{T}$
$\mathrm{H}$

very rare

rare

rare

rather rare

very rare

Ar

A

$\mathrm{T}$

rare

$\begin{array}{cccc}\text { Ar } & \text { A } & \mathrm{T} & \text { rather rare } \\ \text { Ep } & \mathrm{A} & \mathrm{T} & \text { very rare } \\ \mathrm{Ar} & \mathrm{A} & \mathrm{T} & \text { rare } \\ \mathrm{As} & \mathrm{A} & \mathrm{T}(\mathrm{H}) & \text { frequent } \\ \mathrm{Ep} & \mathrm{A} & \mathrm{T} & \text { very rare } \\ \mathrm{Ar} & \mathrm{A} & \mathrm{T} & \text { rather rare } \\ \mathrm{Aw} & \mathrm{A} & \mathrm{T} & \text { rather rare } \\ \mathrm{Aw} & \mathrm{P} & \mathrm{G}(\mathrm{H}) & \text { frequent } \\ \mathrm{Ar} & \mathrm{P} & \mathrm{G} & \text { rather rare } \\ \mathrm{As} & \mathrm{A} & \mathrm{H} & \text { rare } \\ \mathrm{At} & \mathrm{A} & \mathrm{T} & \text { rather rare } \\ \text { As } & \mathrm{A} & \mathrm{T} & \text { rare } \\ \text { Ar } & \mathrm{A} & \mathrm{T} & \text { very rare } \\ \mathrm{Ar} & \mathrm{A} & \mathrm{T} & \text { very common } \\ \text { As } & \mathrm{A} & \mathrm{T}(\mathrm{H}) & \text { very rare } \\ \mathrm{Ar} & \mathrm{A} & \mathrm{T} & \text { rather common }\end{array}$


75. Lepidium ruderale L.

76. Brassica napus L.

77. B. nigra (L.) W. D. J. Koch

78. Sinapis arvensis L.

79. S. alba L.

80. Raphanus raphanistrum L.

$\begin{array}{llcc}\text { Ar } & \text { A } & \mathrm{T} & \text { very rare } \\ \text { Er } & \text { A } & \mathrm{T} & \text { very rare } \\ \text { Er } & \text { A } & \mathrm{T} & \text { very rare } \\ \text { Ar } & \text { A } & \mathrm{T} & \text { rather rare } \\ \text { Er } & \text { A } & \mathrm{T} & \text { very rare } \\ \text { Ar } & \text { A } & \mathrm{T}(\mathrm{H}) & \text { very common }\end{array}$

Crassulaceae

81. Sedum maximum (L.) Hoffm.

Rosaceae

82. Rubus caesius L.

83. Agrimonia eupatoria L.

84. Sanguisorba officinalis L.

85. Geum urbanum L.

86. Potentilla anserina L.

87. P. erecta (L.) Raeusch.

88. $P$. reptans L.

89. P. norvegica $\mathrm{L}$.

90. P. arenaria Borkh.

91. P. collina Wibel

92. P. argentea L. S.str.

93. Alchemilla monticola Opiz

Fabaceae

94. Vicia sepium L.

95. V. grandiflora Scop.

At

$\mathrm{P}$

G

Af

At

Am

Af

Am

Af

Am

Aw

At

Aw

At

Am

Af

Ep

96. V. sativa $\mathrm{L}$.

97. V. angustifolia L.

98. V. hirsuta (L.) S.F. Gray

99. V. tetrasperma (L.) Schreb.

100. V. villosa Roth.

101. V. cracca L.

102. Lathyrus pratensis L.

103. L. tuberosus L.

104. Melilotus alba Medik.

105. M. officinalis (L.) Pall.

106. Medicago falcata L.

107. M. sativa L. s.str.

108. M. lupulina L.

109. Trifolium dubium Sibth.

110. T. campestre Schreb .

111. T. montanum L.

112. T. repens L.

113. T. hybridum L.

114. T. medium L.

115. T. pratense L.

f

t

An

Af

Af

m

t

N




\begin{tabular}{|c|c|c|c|c|}
\hline 116. T. arvense L. & As & A & $\mathrm{T}$ & rather rare \\
\hline 117. Lotus corniculatus L. & $\mathrm{Am}$ & A & $\mathrm{T}$ & rare \\
\hline \multicolumn{5}{|l|}{ Oxalidaceae } \\
\hline 118. Oxalis stricta L. & Ep & $\mathrm{P}$ & $\mathrm{H}$ & frequent \\
\hline \multicolumn{5}{|l|}{ Geraniaceae } \\
\hline 119. Geranium pratense L. & Am & $\mathrm{P}$ & $\mathrm{H}$ & very rare \\
\hline 120. G. dissectum L. & $\mathrm{Ar}$ & A & $\mathrm{T}$ & very rare \\
\hline 121. G. pyrenaicum Burm. F. & Ep & $\mathrm{P}$ & $\mathrm{H}$ & very rare \\
\hline 122. G. pusillum Burm. F. ex L. & $\mathrm{Ar}$ & A & $\mathrm{T}$ & rather common \\
\hline 123. Erodium cicutarium (L.) L'Hér. & $\mathrm{Ar}$ & A & $\mathrm{T}(\mathrm{H})$ & common \\
\hline \multicolumn{5}{|l|}{ Euphorbiaceae } \\
\hline 124. Euphorbia helioscopia L. & $\mathrm{Ar}$ & A & $\mathrm{T}$ & rather rare \\
\hline 125. E. peplus L. & $\mathrm{Ar}$ & A & $\mathrm{T}$ & rare \\
\hline 126. E. cyparissias L. & At & $\mathrm{P}$ & $\mathrm{G}(\mathrm{H})$ & very rare \\
\hline 127. E. esula L. & At & $\mathrm{P}$ & G & very rare \\
\hline \multicolumn{5}{|l|}{ Malvaceae } \\
\hline 128. Malva alcea $\mathrm{L}$. & Ar & A & $\mathrm{H}$ & very rare \\
\hline 129. M. pusilla $\mathrm{Sm}$. & Ar & A & $\mathrm{H}$ & rare \\
\hline 130. M. neglecta Wallr. & $\mathrm{Ar}$ & A & $\mathrm{T}$ & rare \\
\hline 131. M. sylvestris L. & $\mathrm{Ar}$ & $\mathrm{A}(\mathrm{P})$ & $\mathrm{H}$ & very rare \\
\hline \multicolumn{5}{|l|}{ Clusiaceae } \\
\hline 132. Hypericum humifusum $\mathrm{L}$. & As & $\mathrm{P}$ & $\mathrm{T}(\mathrm{H})$ & rather rare \\
\hline 133. H. perforatum L. & $\mathrm{Am}$ & $\mathrm{P}$ & $\mathrm{H}$ & rare \\
\hline \multicolumn{5}{|l|}{ Violaceae } \\
\hline 134. Viola arvensis Murray & $\mathrm{Ar}$ & A & $\mathrm{T}$ & very common \\
\hline 135. V. tricolor L. s.str. & As & A & $\mathrm{T}$ & very rare \\
\hline \multicolumn{5}{|l|}{ Cucurbitaceae } \\
\hline 136. Echinocystis lobata (F. Michx.) Torr. et A. Gray & Ep & A & $\mathrm{T}$ & very rare \\
\hline \multicolumn{5}{|l|}{ Lythraceae } \\
\hline 137. Peplis portula L. & Aw & A & $\mathrm{T}$ & rare \\
\hline 138. Lythrum salicaria L. & Am & $\mathrm{P}$ & $\mathrm{H}$ & rare \\
\hline \multicolumn{5}{|l|}{ Onagraceae } \\
\hline 139. Oenothera biennis L. & As & A & $\mathrm{T}$ & very rare \\
\hline 140. Chamaenerion angustifolium (L.) Scop. & As & $\mathrm{P}$ & $\mathrm{H}$ & very rare \\
\hline 141. Epilobium parviflorum Schreb. & Aw & $\mathrm{P}$ & $\mathrm{H}$ & very rare \\
\hline 142. E. roseum Schreb. & Aw & $\mathrm{P}$ & $\mathrm{H}$ & rare \\
\hline \multicolumn{5}{|l|}{ Apiaceae } \\
\hline 143. Eryngium planum L. & As & $\mathrm{P}$ & $\mathrm{H}$ & very rare \\
\hline 144. Anthriscus sylvestris (L.) Hoffm. & Af & $\mathrm{P}$ & $\mathrm{H}$ & very rare \\
\hline 145. Pimpinella saxifraga L. & At & $\mathrm{P}$ & $\mathrm{H}$ & rare \\
\hline 146. Aegopodium podagraria L. & At & $\mathrm{P}$ & $\mathrm{H}$ & very rare \\
\hline 147. Aethusa cynapium L. & $\mathrm{Ar}$ & A & $\mathrm{T}$ & rare \\
\hline 148. Carum carvi L. & Am & A & $\mathrm{T}$ & very rare \\
\hline 149. Pastinaca sativa L. & As & $\mathrm{P}$ & $\mathrm{H}$ & very rare \\
\hline
\end{tabular}




\begin{tabular}{|c|c|c|c|c|}
\hline 150. Heracleum sphondylium L. & $\mathrm{Am}$ & $\mathrm{P}$ & $\mathrm{H}$ & very rare \\
\hline 151. Torilis japonica (Houtt) DC. & Af & A & $\mathrm{T}$ & very rare \\
\hline 152. Daucus carota L. & $\mathrm{Ar}$ & A & $\mathrm{T}$ & rather rare \\
\hline \multicolumn{5}{|l|}{ Primulaceae } \\
\hline 153. Lysimachia nummularia L. & $\mathrm{Am}$ & $\mathrm{P}$ & G & rare \\
\hline 154. L. vulgaris L. & Am & $\mathrm{P}$ & $\mathrm{G}(\mathrm{H})$ & very rare \\
\hline 155. Centunculus minimus L. & $\mathrm{Ar}$ & A & $\mathrm{T}$ & rather rare \\
\hline 156. Anagallis arvensis L. & $\mathrm{Ar}$ & A & $\mathrm{T}$ & frequent \\
\hline \multicolumn{5}{|l|}{ Gentianaceae } \\
\hline 157. Centaurium pulchellum (Sw.) Druce & Am & A & $\mathrm{T}$ & rare \\
\hline \multicolumn{5}{|l|}{ Rubiaceae } \\
\hline 158. Galium palustre L. & Aw & $\mathrm{P}$ & $\mathrm{H}$ & very rare \\
\hline 159. G. aparine L. & Af & A & $\mathrm{T}$ & common \\
\hline 160. G. spurium L. & $\mathrm{Ar}$ & A & $\mathrm{T}$ & very rare \\
\hline 161. G. boreale L. & Af & $\mathrm{P}$ & G & very rare \\
\hline 162. G. verum L. s. str. & $\mathrm{Am}$ & $\mathrm{P}$ & G & rare \\
\hline 163. G. mollugo L. s.str. & $\mathrm{Am}$ & $\mathrm{P}$ & G & rare \\
\hline
\end{tabular}

Convolvulaceae

164. Convolvulus arvensis L.

At

$\mathrm{P}$

G

very common

Boraginaceae

165. Lithospermum arvense L.

166. Echium vulgare $\mathrm{L}$.

167. Symphytum officinale L.

168. Anchusa officinalis L.

169. A. arvensis (L.) M. Bieb.

170. Myosotis arvensis (L. Hill.

171. M. stricta Link ex Roem. et Schult.

Ar

At

Aw (m)

At

Ar

Ar

As

172. Cynoglossum officinalis L.

\section{Lamiaceae}

173. Galeopsis ladanum L.

174. G. speciosa Mill.

175. G. pubescens Besser

176. G. tetrahit L.

177. G. bifida Boenn.

178. Lamium album $\mathrm{L}$.

179. L. ampexicaule L.

180. L. purpureum L.

181. Ballota nigra L.

182. Stachys annua L.

183. S. palustris L.

184. Glechoma hederacea L.

185. Prunella vulgaris L.

186. Thymus serpyllum L. M.Fr.

187. Lycopus europeus L.

As

As

Ar

Af

Af

Af

Af

Ar

Ar

Ar

Ar

Ar

Am (w)

Am

Am

As

Aw
A

A

P

A

A

A

A

A

A

A

A

A

A

P

A

A

$\mathrm{P}$

A

$\mathrm{P}$

$\mathrm{P}$

$\mathrm{P}$

$\mathrm{P}$

$\mathrm{P}$

$\mathrm{T}$
$\mathrm{H}$
$\mathrm{G}$
$\mathrm{T}$
$\mathrm{T}$
$\mathrm{T}(\mathrm{H})$
$\mathrm{T}$
$\mathrm{T}(\mathrm{H})$

rare very rare rare rare rare very common rather rare very rare

rather rare rare rather rare rather common common very rare rather rare frequent very rare very rare rather common G $\mathrm{H}$ $\mathrm{H}$ $\mathrm{H}$ $\mathrm{H}$ 


\begin{tabular}{|c|c|c|c|c|}
\hline 188. Mentha arvensis $\mathrm{L}$. & Aw & $\mathrm{P}$ & G & common \\
\hline 189. Elsholtzia ciliata (Thunb.) Hyl. & Ep & A & $\mathrm{T}$ & very rare \\
\hline \multicolumn{5}{|l|}{ Solanaceae } \\
\hline 190. Solanum dulcamara L. & Aw & $\mathrm{P}$ & $\mathrm{H}$ & very rare \\
\hline 191. S. nigrum L. em. Mill. & $\mathrm{Ar}$ & A & $\mathrm{T}$ & rare \\
\hline 192. Datura stramonium L. & Ep & A & $\mathrm{T}$ & very rare \\
\hline \multicolumn{5}{|l|}{ Scrophulariaceae } \\
\hline 193. Verbascum nigrum $\mathrm{L}$. & Af & A & $\mathrm{H}$ & very rare \\
\hline 194. Scrophularia nodosa L. & Af & $\mathrm{P}$ & $\mathrm{H}$ & very rare \\
\hline 195. Chaenorhinum minus (L.) Lange & At & A & $\mathrm{T}$ & rare \\
\hline 196. Linaria vulgaris Mill. & As & $\mathrm{P}$ & G & rare \\
\hline 197. Veronica serpyllifolia L. & Am & $\mathrm{P}$ & $\mathrm{H}$ & rather rare \\
\hline 198. V. triphyllos L. & $\mathrm{Ar}$ & A & $\mathrm{T}$ & very rare \\
\hline 199. V. arvensis L. & $\mathrm{Ar}$ & A & $\mathrm{T}$ & very common \\
\hline 200. V. verna $\mathrm{L}$. & As & A & $\mathrm{T}$ & very rare \\
\hline 201. V dillenii Crantz & As & A & $\mathrm{T}$ & rare \\
\hline 202. V. hederifolia L. s.str. & Af & A & $\mathrm{T}$ & very rare \\
\hline 203. V. persica Poir. & Ep & A & $\mathrm{T}$ & frequent \\
\hline 204. V. agrestis L. & $\mathrm{Ar}$ & A & $\mathrm{T}$ & rather rare \\
\hline 205. V.polita Fr. & $\mathrm{Ar}$ & A & $\mathrm{T}$ & rare \\
\hline 206. V. opaca Fr. & $\mathrm{Ar}$ & A & $\mathrm{T}$ & very rare \\
\hline 207. V. chamaedrys L. s.str. & Am & $\mathrm{P}$ & $\mathrm{H}$ & very rare \\
\hline 208. Odontites verna (Bellardi) Dummort. & $\mathrm{Ar}$ & A & $\mathrm{T}$ & very rare \\
\hline 209. O. serotina (Lam.) Rchb. s.str. & Am & A & $\mathrm{T}$ & rather rare \\
\hline 210. Rhinanthus serotinus (Schönh.) Oborný & Ar & A & $\mathrm{T}$ & frequent \\
\hline \multicolumn{5}{|l|}{ Plantaginaceae } \\
\hline 211. Plantago media L. s.str. & Am & $\mathrm{P}$ & $\mathrm{H}$ & very rare \\
\hline 212. P. major L. & Af & $\mathrm{P}$ & $\mathrm{H}$ & rather common \\
\hline 213. P. intermedia Gilib. & Aw & $\mathrm{P}$ & $\mathrm{H}$ & common \\
\hline 214. P. lanceolata L. & $\mathrm{Am}$ & $\mathrm{P}$ & $\mathrm{H}$ & frequent \\
\hline \multicolumn{5}{|l|}{ Dipsacaceae } \\
\hline 215. Knautia arvensis (L.) J.M. Coult. & $\mathrm{Am}$ & $\mathrm{P}$ & $\mathrm{H}$ & rather rare \\
\hline \multicolumn{5}{|l|}{ Campanulaceae } \\
\hline 216. Campanula patula L. s.str. & Am & $\mathrm{P}$ & $\mathrm{H}$ & very rare \\
\hline 217. C. rapunculoides L. & At & $\mathrm{P}$ & $\mathrm{G}(\mathrm{H})$ & very rare \\
\hline 218. Jasione montana L. & As & A & $\mathrm{H}$ & rare \\
\hline \multicolumn{5}{|l|}{ Asteraceae } \\
\hline 219. Eupatorium canabinum L. & Aw & $\mathrm{P}$ & $\mathrm{H}$ & very rare \\
\hline 220. Erigeron acris L. & As & A & $\mathrm{T}(\mathrm{H})$ & rather rare \\
\hline 221. Conyza canadensis (L.) Cronquist & Ep & A & $\mathrm{T}$ & common \\
\hline 222. Filago minima (Sm.) Pers. & As & A & $\mathrm{T}$ & very rare \\
\hline 223. Gnaphalium sylvaticum L. & Af & $\mathrm{P}$ & $\mathrm{H}$ & very rare \\
\hline 224. G. uliginosum L. & Aw & A & $\mathrm{T}$ & common \\
\hline 225. Helichrysum arenarium (L.) Moench & As & A & $\mathrm{H}$ & very rare \\
\hline
\end{tabular}


226. Inula britannica L.

227. Bidens tripartita L.

228. Helianthus annuus L.

229. H. tuberosus L.

230. Galinsoga parviflora Cav.

231. G. ciliata (Raf.) S.F.Blake

238. Tanacetum vulgare $\mathrm{L}$.

239. Artemisia absinthium L.

$\mathrm{Ar}$

Am (w)

241. A. campestris $\mathrm{L}$

241. A. campestris L.

242. Tussilago farfara L.

243. Senecio vulgaris L.

244. S. vernalis Waldst. et Kit.

245. Arctium lappa L.

246. Carduus acanthoides L.

247. C. crispus L.

248. Cirsium vulgare (Savi) Ten.

249. C. arvense (L.) Scop.

250. Centaurea cyanus L.

251. C. stoebe L.

252. C. jacea $\mathrm{L}$.

253. Cichorium intybus L.

254. Arnoseris minima (L.) Schweigg. et Körte

255. Hypochoeris radicata $\mathrm{L}$.

256. H. glabra L.

257. Leontodon autumnalis L.

258. Tragopogon dubius Scop.

259. Sonchus oleraceus L.

260. S. asper (L.) Hill

261. S. arvensis L. subsp. arvensis

262. Lactuca serriola L.

263. Taraxacum officinale F.H. Wigg.

264. Lapsana communis L. s. str.

265. Crepis tectorum $\mathrm{L}$.

266. C. capillaris (L.) Wallr.

267. Hieracium pilosella $\mathrm{L}$.

268. H. umbellatum L.

\section{Liliaceae}

Aw

Ar

Ep

Af

Ar

Ep

Af

Af

Ar

At

Am

$\mathrm{Ar}$

As

Am

As

Am

As

Ar

Ar

Aw

$\mathrm{Ar}$

Am

Af

As

Am

As

Am

Aw
Aw
Er
Ep
Ep
Ar
Am
Am
Ar
Ar
Ep

\begin{tabular}{|c|c|}
\hline $\mathrm{H}$ & very rare \\
\hline $\mathrm{T}$ & rather rare \\
\hline $\mathrm{T}$ & very rare \\
\hline G & very rare \\
\hline $\mathrm{T}$ & common \\
\hline $\mathrm{T}$ & frequent \\
\hline $\mathrm{T}$ & very common \\
\hline $\mathrm{H}$ & very rare \\
\hline $\mathrm{H}$ & common \\
\hline $\mathrm{T}(\mathrm{H})$ & very common \\
\hline $\mathrm{T}$ & rare \\
\hline $\mathrm{T}$ & rather rare \\
\hline $\mathrm{H}$ & rare \\
\hline $\mathrm{H}$ & rare \\
\hline $\mathrm{H}$ & common \\
\hline $\mathrm{H}$ & rare \\
\hline G & very rare \\
\hline $\mathrm{T}(\mathrm{H})$ & rare \\
\hline $\mathrm{T}(\mathrm{H})$ & very rare \\
\hline $\mathrm{H}$ & rare \\
\hline $\mathrm{H}$ & rare \\
\hline $\mathrm{H}$ & very rare \\
\hline $\mathrm{H}$ & very rare \\
\hline G & very common \\
\hline $\mathrm{T}$ & very common \\
\hline $\mathrm{H}$ & very rare \\
\hline $\mathrm{H}$ & rare \\
\hline G & rare \\
\hline $\mathrm{T}$ & frequent \\
\hline $\mathrm{H}$ & rare \\
\hline $\mathrm{T}$ & very rare \\
\hline $\mathrm{H}$ & rare \\
\hline $\mathrm{T}$ & very rare \\
\hline $\mathrm{T}(\mathrm{H})$ & rather rare \\
\hline $\mathrm{T}$ & frequent \\
\hline $\mathrm{G}(\mathrm{H})$ & common \\
\hline $\mathrm{H}$ & rare \\
\hline $\mathrm{H}$ & common \\
\hline $\mathrm{T}(\mathrm{H})$ & rather rare \\
\hline $\mathrm{T}$ & rather rare \\
\hline $\mathrm{T}$ & rare \\
\hline $\mathrm{H}$ & rare \\
\hline $\mathrm{H}$ & very rare \\
\hline
\end{tabular}

As

At

P

G

rare 


\begin{tabular}{|c|c|c|c|c|}
\hline Juncaceae & & & & \\
\hline 270. Juncus capitatus Weigel & Aw & A & $\mathrm{T}$ & rare \\
\hline 271. J. bufonius L. & Aw & A & $\mathrm{T}$ & frequent \\
\hline \multicolumn{5}{|l|}{ Poaceae } \\
\hline 272. Festuca pratensis Huds. & $\mathrm{Am}$ & $\mathrm{P}$ & $\mathrm{H}$ & very rare \\
\hline 273. F. rubra L. s. str. & $\mathrm{Am}$ & $\mathrm{P}$ & $\mathrm{H}$ & very rare \\
\hline 274. F. ovina L. s. str. & Af & $\mathrm{P}$ & $\mathrm{H}$ & very rare \\
\hline 275. Lolium perenne L. & Am & $\mathrm{P}$ & $\mathrm{H}$ & rare \\
\hline 276. Poa annua L. & $\mathrm{Am}$ & A & $\mathrm{T}(\mathrm{H})$ & frequent \\
\hline 277. P. pratensis L. s. str. & Am & $\mathrm{P}$ & $\mathrm{H}$ & rare \\
\hline 278. P palustris L. & Aw & $\mathrm{P}$ & $\mathrm{H}$ & very rare \\
\hline 279. Dactylis glomerata L. & Am & $\mathrm{P}$ & $\mathrm{H}$ & rare \\
\hline 280. Apera spica-venti (L.) P. Beauv. & $\mathrm{Ar}$ & A & $\mathrm{T}$ & very common \\
\hline 281. Bromus inermis Leyss. & At & $\mathrm{P}$ & $\mathrm{H}$ & very rare \\
\hline 282. B. secalinus L. & Ar & A & $\mathrm{T}$ & frequent \\
\hline 283. B. hordeaceus L. & Am & $\mathrm{P}$ & $\mathrm{H}$ & very rare \\
\hline 284. Elymus repens (L.) Gould & Aw & $\mathrm{P}$ & $\mathrm{G}$ & very common \\
\hline 285. Avena strigosa Schreb. & $\mathrm{Ar}$ & A & $\mathrm{T}$ & rather common \\
\hline 286. A. fatua L. & $\mathrm{Ar}$ & A & $\mathrm{T}$ & frequent \\
\hline 287. Anthoxanthum aristatum Boiss. & Ep & A & $\mathrm{T}$ & rare \\
\hline 288. Holcus lanatus L. & Am & $\mathrm{P}$ & $\mathrm{H}$ & rare \\
\hline 289. H. mollis L. & Af & $\mathrm{P}$ & $\mathrm{H}$ & rare \\
\hline 290. Corynephorus canescens (L.) P. Beauv. & As & $\mathrm{P}$ & $\mathrm{H}$ & very rare \\
\hline 291. Agrostis gigantea Roth & Am & $\mathrm{P}$ & $\mathrm{H}$ & rare \\
\hline 292. A. stolonifera L. & Am & $\mathrm{P}$ & $\mathrm{H}$ & common \\
\hline 293. Phleum pratense L. & Am & $\mathrm{P}$ & $\mathrm{H}$ & rare \\
\hline 294. Alopecurus geniculatus L. & $\mathrm{Am}$ & $\mathrm{P}$ & $\mathrm{G}(\mathrm{H})$ & rare \\
\hline 295. A. myosuroides Huds. & Am & $\mathrm{P}$ & $\mathrm{G}(\mathrm{H})$ & very rare \\
\hline 296. A. pratensis L. & $\mathrm{Am}$ & $\mathrm{P}$ & $\mathrm{G}(\mathrm{H})$ & very rare \\
\hline 297. Phalaris arundinacea L. & Aw & $\mathrm{P}$ & $\mathrm{H}$ & very rare \\
\hline 298. Phragmites australis (Cav.) Trin. ex Steud. & Aw & $\mathrm{P}$ & $\mathrm{G}$ & rare \\
\hline 299. Nardus stricta L. & As & $\mathrm{P}$ & $\mathrm{H}$ & very rare \\
\hline 300. Echinochloa crus-galli (L.) P. Beauv. & $\mathrm{Ar}$ & A & $\mathrm{T}$ & very common \\
\hline 301. Digitaria ischaemum (Schreb.) H.L. Mühl. & $\mathrm{Ar}$ & A & $\mathrm{T}$ & rather common \\
\hline 302. D. sanguinalis (L.) Scop. & Ar & A & $\mathrm{T}$ & very rare \\
\hline 303. Setaria pumila (Poir.) Roem et Schult. & $\mathrm{Ar}$ & A & $\mathrm{T}$ & rather common \\
\hline 304. S. viridis (L.) P. Beauv . & Ar & A & $\mathrm{T}$ & frequent \\
\hline \multicolumn{5}{|l|}{ Cyperaceae } \\
\hline 305. Carex hirta L. & $\mathrm{Am}$ & $\mathrm{P}$ & $\mathrm{G}$ & very rare \\
\hline
\end{tabular}

Comments: Am - apophytes of meadow habitats, Af - apophytes of forest, As - apophytes of dunes and sands, At - apophytes of thermophilous grasslands, Aw - apophytes of waterside and marshy habitat, Ar - archeophytes, Ep - epecophytes, Er ergasiophytes, A - annual and biennial species, $\mathrm{P}$ - perennial species, $\mathrm{T}$ - therophytes, $\mathrm{H}$ - hemicryptophytes, $\mathrm{Ch}-\mathrm{chamaephytes,}$ $\mathrm{G}-$ geophytes. 


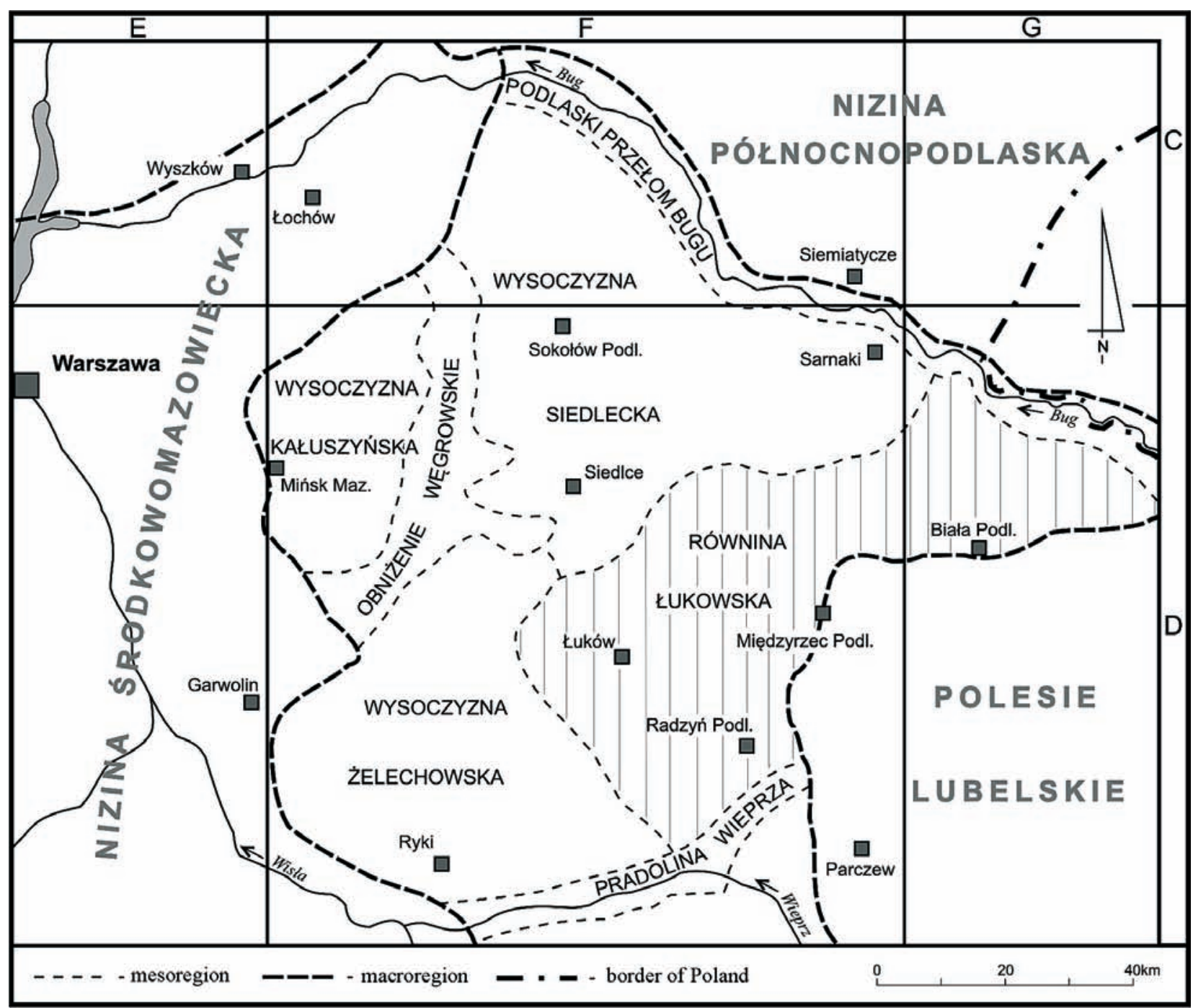

Fig. 1. Location of the Łuków Plain against a background of physical-geographic division of Poland

\section{Flora}

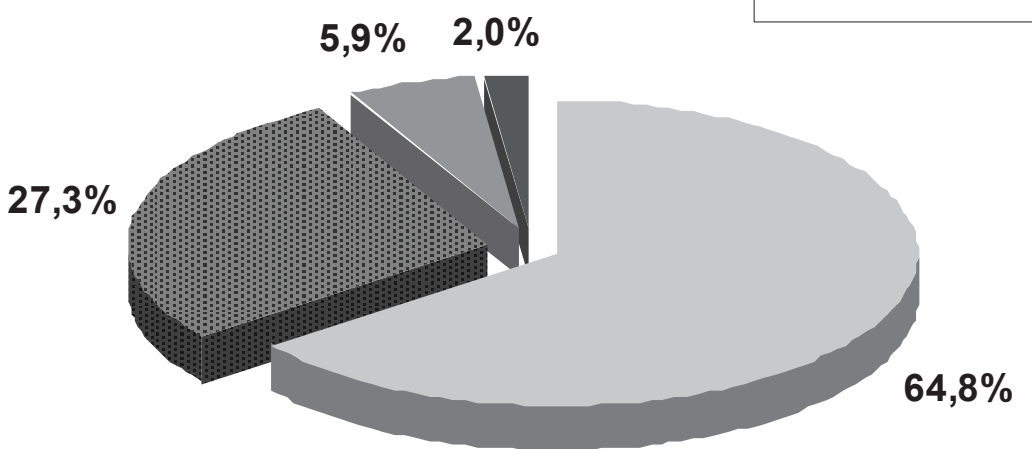

Fig. 2. Proportions of geographical-historical groups in the segetal flora of the Łuków Plain 1 - apophytes, 2 - archeophytes, 3 - epecophytes, 4 - ergasiophytes 


\section{Apophytes}

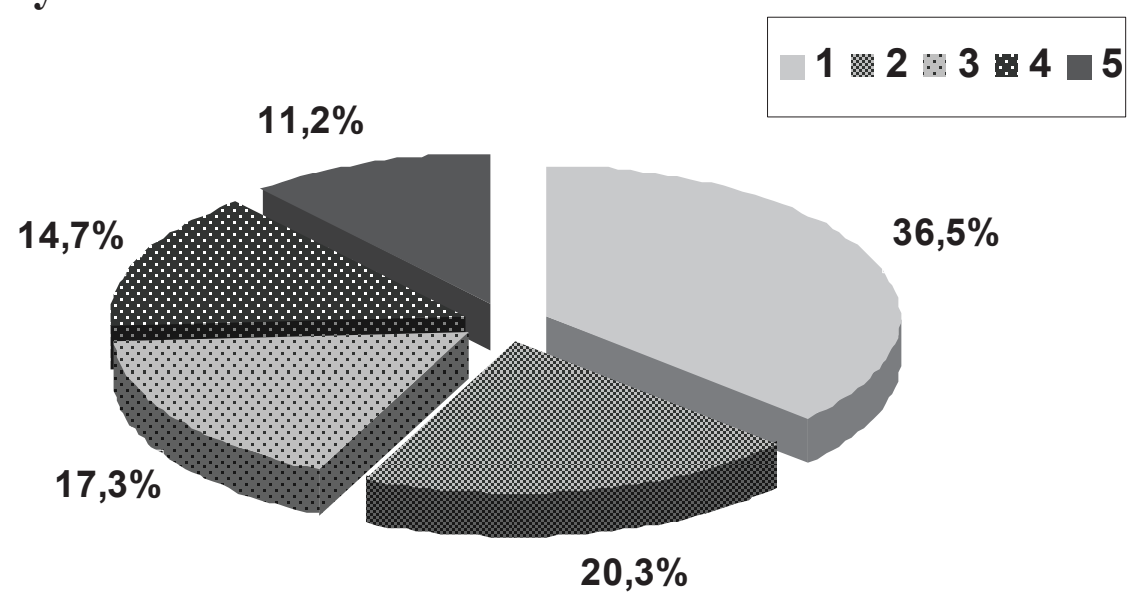

Fig. 3. Origin of apophytes of the segetal flora of the Łuków Plain 1 - meadows habitats, 2 - waterside and marshy habitats, 3 - dunes and sands, 4 - forest and bushes, 5 - thermophilous grasslands

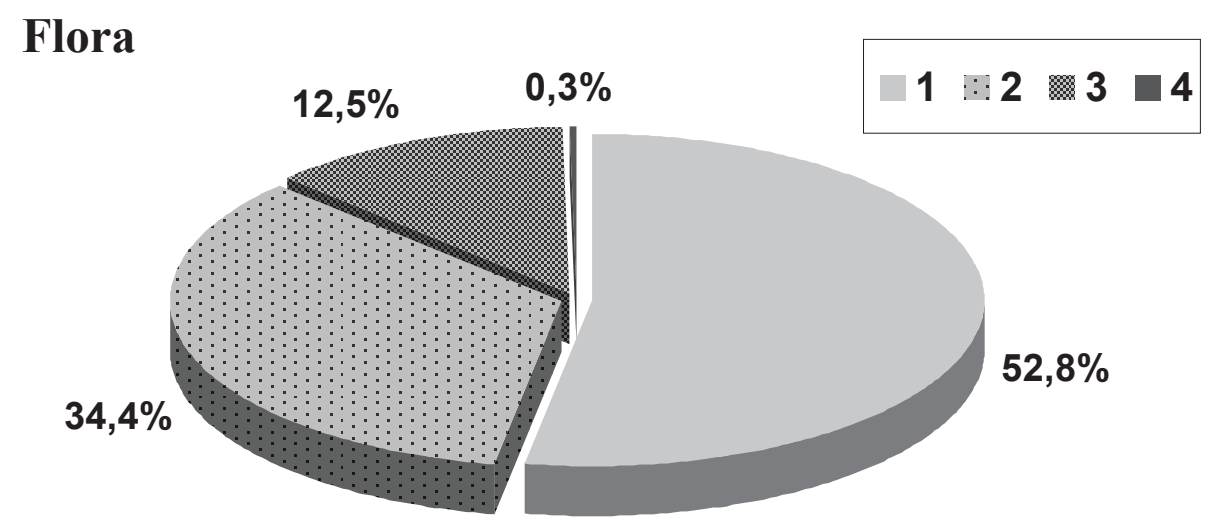

Antropophythes

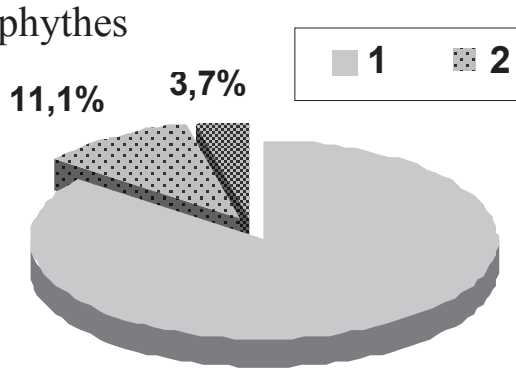

$85,2 \%$

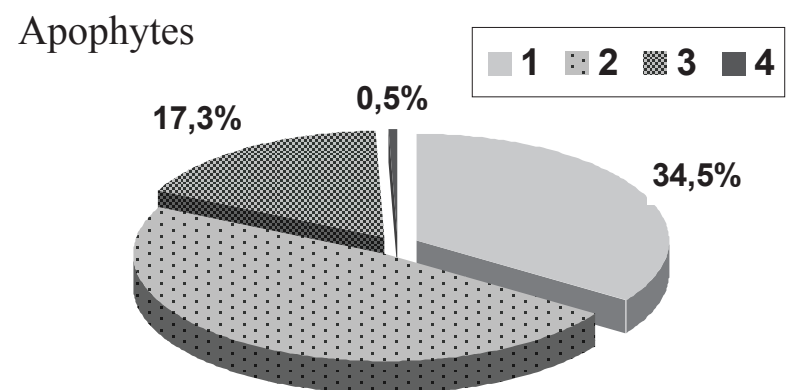

$47.7 \%$

Fig. 4. Proportions of life forms in the segetal flora of the Euków Plain 1 - therophytes, 2 - hemicryptophytes, 3 - geophytes, 4 - chamaephytes 


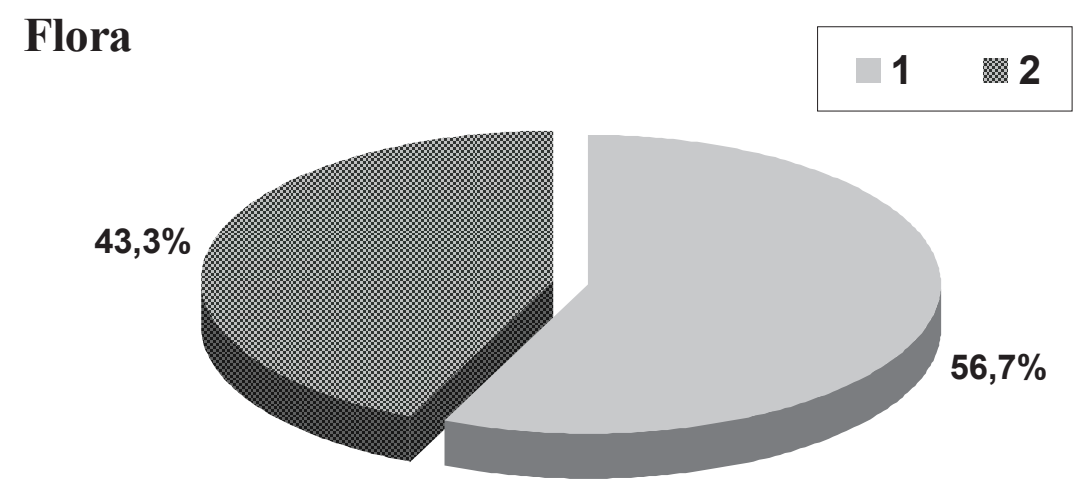

Antropophythes

Apophytes

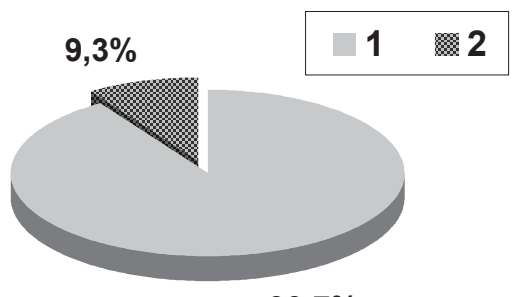

$90,7 \%$

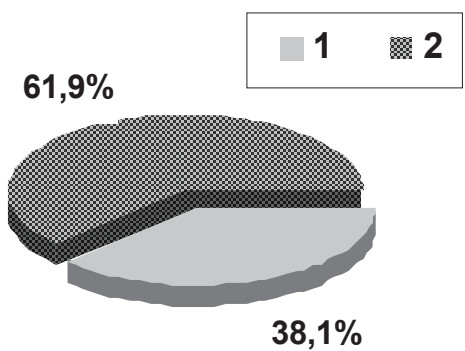

Fig. 5. Life span of species in the segetal flora of the Łuków Plain 1 - annual and biennial species, 2 - perennial species

\section{DISCUSSION}

The segetal flora of the Łuków Plain is abundant and diverse. When it comes to the number of species, it is far above the florae of Żuławy Wiślane Region (H o ł d y ń s k i , 1991) and Wielkopolska Region (L a t ow ski et al. 1979) or the area of arable lands of Bolimowska Primeval Forest (W a r c h o li ń s k a, 1988 ) and others. However, the number of species recorded in the investigated area is slightly lower than in the segetal florae of the neighbouring mesoregions: considerably smaller than that of Podlaski Przełom Bugu - 346 species ( $\mathrm{Skrzy}$ c z y ńska and Rzymow s k a, 2001), and similarly compared to the Siedlce Upland area - 320 species ( $\mathrm{S} \mathrm{k} \mathrm{r} \mathrm{z} \mathrm{y} \mathrm{c} \mathrm{z} \mathrm{y} \mathrm{ń} \mathrm{s} \mathrm{k} \mathrm{a,}$ 1994). The floristic abundance of the area under study stems not only from its considerable size but also from the diversity of habitats, the patch-like system of small fields, and relatively traditional agriculture. The influence of similar factors on segetal florae has also been mentioned by: Korniak (1992), Skrzyczyńska and Rzymowska (2001), Warcholińs k a (1991), and others. Most of the above-mentioned factors also result in the prevalence of apophytes over alien species. These correlations are confirmed by investigations from other regions of Poland ( $\mathrm{K}$ or n iak, 1992; B o man ow ska, 2006; W a r choli ń s k a , 1991). The significance of the considerable contribution of light and acid soils in the Łuków Plain is also undeniable, as they make a favourable habitat for native species that occur here abundantly, especially those from sand habitats and dunes. The phenomenon of dominance of apophytes was observed in the Masovian Region by Wó j c i k (1968), who is of the opinion that in these habitats apophytes successfully compete with specialised, typical field weeds, particularly archaeophytes.

The extensive method of farming and habitat characteristics also influence the biological spectrum and stability of the species. The proportion of therophytes is slightly over $50 \%$, with a substantial representation of hemicryptophytes and geophytes. The percentage structure of life-forms is similar to that observed in Podlachian Bug River Gap (S k r z y c z y ń ska and Rzymowska, 2001) and north-eastern Poland (Korniak, 1992). S z otkowski (1989) explains the decreasing rate of hemicryptophytes by the effect of human activity defined as changes in agro-technology. A relatively small prevalence of annuals and biennials over perennials was observed in the investigated area. This fact indirectly stems from the dominance of apophytes and a considerable representation of hemicryptophytes and geophytes, which is caused by the same factors that contribute to the prevalence of apophytes over anthropophytes. 
In the flora in question, the most numerously represented families are Asteraceae and Poaceae. The same tendency is observed not only in the segetal florae of different regions of Poland ( $\mathrm{R} \mathrm{a} \mathrm{t} \mathrm{u} \mathrm{s} \mathrm{z} \mathrm{n} \mathrm{i} \mathrm{a} \mathrm{k} \mathrm{and}$ S o b i s z, 2006; S k r z y c z y ń s k a, 1994; B o m an o w s k a, 2006), but also in the florae of other European countries, both in the south and north of Europe (Glemnitz et al. 2000).

Sub-Atlantic species occur in the Łuków Plain with different frequency. Arnoseris minima is a frequent species; though, it is less frequent in the east of the mesoregion, whereas Teesdalea nudicaulis is a very rare species. Stands of the latter species in the area under study were recorded by $\mathrm{C}$ i o se $\mathrm{k}$ and $\mathrm{Skrzy}$ c z yńska (1989) and Ci os ek et al. (1997). The narrow ecological amplitude and the decrease in the area of poor, acidic habitats (afforestation, fields lying fallow) result in regression of these species. Another reason for their disappearance is the high fertilization level and use of herbicides. The above mentioned species are considered to be rare components of the flora that are threatened with extinction in many regions of Poland and in Europe (Fija k k ow ski and N y c z, 1998; U r bis z et al. 1998; S i c iń s ki, 1998; K u bat and Kaplan, 2001, Prasse et al. 2001; Cheffings and Farre11, 2005; Pinke et al. 2006). Anthoxanthum aristatum also belongs to rare species. When studying rare species in the South Podlachian Lowland, Ci o s e k and S krzyczyńska (1989) did not record stands of this species in the Łuków Plain.

The characteristic feature of the investigated area is the occurrence of abundant populations of rare speirochoric species that are becoming extinct in Poland, such as Agrostemma githago, Bromus secalinus, and Avena strigosa. These species are in regression in Poland as a result of use of certified seed material (W a r cholińsk a , 1981, 2002; Frey, 1989; Żukowski and Jackowiak, 1995; AniołKwiatkow ska, 1998; S i c iński, 1998; No$\mathrm{w}$ a $\mathrm{k}$ et al. 2003). The persistence of these species in the agrocenoses of the Łuków Plain arises from traditional farming methods, particularly from the growers' use of their own badly-cleaned sowing material, which results from poor financial conditions of many farms in the region. The connection between rare speirochoric species and extensive agriculture has been mentioned by Kornaś $(1972,1987)$, Hołdyński and W oźn i a k (1994), H o c hół (1990), and others.

\section{REFERENCES}

Anioł-Kwiatkowska J., 1974. Flora i zbiorowiska synantropijne Legnicy, Lubina i Polkowic / La flore et les groupe synanthropiques de Legnica, Lubin et Polkowice
Acta Univ. Wratisl., Prace Bot. 229 (19): 1-152. (in Polish)

A nioł-Kwiatkowska J., 1998. Ginące i zagrożone gatunki segetalne na Wale Trzebnickim / Endangered and rare segetal species in the Macroregion Trzebnica Hills. Acta Univ. Lodz. Folia bot. 13: 169-176. (in Polish)

Bomanowska A., 2006. Wybrane cechy flory segetalnej Kampinowskiego Parku Narodowego/ Some features of the segetal flora of the Kampinos National Park. Pam. Puław., IUNG Puławy, 143: 27-35. (in Polish)

Ciosek M. T., Celińska E., Krechowski J., 1997. Waloryzacja szaty roślinnej gminy Adamów (woj. siedleckie) / The evaluation of vegetation in Adamów commune (Siedlce Province). Zesz. Nauk. WSR-P w Siedlcach, 51: 87-114. (in Polish)

Ciosek M.T., Pikulski M., 1997. Notatki florystyczne z okolic Osin (Równina Łukowska) / Floristic notes from the environs of Osiny (Łuków Plain). Zesz. Nauk. WSR-P w Siedlcach, 51: 205-213. (in Polish)

Ciosek M.T., Skrzyczyńska J., 1989. Rzadkie gatunki chwastów polnych gleb lekkich na Nizinie Południowopodlaskiej i terenach przyległych / Rare species of field weeds on the light soils of South Podlasie Lowland and the neighbouring regions. Zesz. Nauk. WSR-P w Siedlcach, ser. Rolnictwo, 20: 123-146. (in Polish)

Cheffings, C.M., Farrell, L. (Eds), Dines, T.D., Jones, R.A., Leach, S.J., Mckean, D.R., Pearman, D.A., Preston, C.D., Rumsey, F.J., Taylor, I., 2005. The Vascular Plant Red Data List for Great Britain. Joint Nature Conservation Committee, Peterborough, 7: 1-116.

Fijałkowski D., 1994. Flora roślin naczyniowych Lubelszczyzny. / The flora of vascular plants of the Lublin region. Środowisko Przyrodnicze Lubelszczyzny LTN Lublin, 1: 389. (in Polish)

Fijałkowski D., Nycz B., 1998. Zagrożone gatunki roślin segetalnych na Lubelszczyźnie / Threatened species of segetal plants in the Lublin region. Acta Univ. Lodz., Folia bot. 13: 199-208. (in Polish)

Frey L., 1989. Rozmieszczenie Avena strigosa Schreb. w Polsce / The distribution of Avena strigosa Schreb. in Poland. Fragm. Flor. et Geobot. 34(1-2): 43-51. (in Polish)

Glemnitz M., Czimber G., Radics L., Hoffman $n$ J., 2000. Weed flora composition along a north-south climate gradient in Europe. Acta Agronomica Ováriensis, 42: 155-169.

Głowacki Z., Rogowiec M., Kuć D., 1995. Charakterystyka rolnicza mezoregionów. Równina Łukowska. W: H. Kot (red.) Przyroda województwa siedleckiego / Agricultural characteristics of mesoregions. The $\mathrm{Eu}-$ ków Plain. [In]: H. Kot (ed.) The nature of the Siedlce Voivodeship. Zakład Badań Ekologicznych EKOS: 5876. (in Polish)

Hochół T., 1990. Zbiorowiska chwastów segetalnych w dolinie rzeki Łososiny w Beskidzie Wyspowym. Cz. II. Zachwaszczenie upraw roślin zbożowych / Segetal 
weed communities in the Łososina Valley in Beskid Wyspowy. Part II. Weed infestation of cereal crops. Zesz. Nauk. AR w Krakowie. 247(29): 77-92. (in Polish)

Hołdyński Cz ., 1991. Flora segetalna, zróżnicowanie florystyczno-ekologiczne i przemiany szaty roślinnej pól uprawnych w aktualnych warunkach agroekologicznych Żuław Wiślanych / Segetal flora, floristic and ecological differentiation and changes in the plant cover of cultivated fields in current agroecological conditions of Żuławy Wislane. Acta Acad. Agricult. Tech. Olst. Agricult. 51, Suppl. B: 3-50. (in Polish)

Hołdyński Cz., Woźniak M., 1994. Speriochoria na tle aktualnego zachwaszczenia pszenicy ozimej na $\dot{Z} u-$ ławach Wiślanych. Mat. XVII Kraj. Kon. Nauk.,, Przyczyny i źródła zachwaszczenia"/ Speirochory against the background of the current weed infestation of winter wheat in Żuławy Wiślane. Proceedings of the 17th National Scientific Conference "Causes and sources of weed infestation" AR-T Olsztyn: 13-20. (in Polish)

Kondracki J ., 2002. Geografia regionalna Polski / The regional geography of Poland s. 441. Państwowe Wydawnictwo Naukowe, Warszawa. (in Polish)

Kornaś J., 1968. Geograficzno-historyczna klasyfikacja roślin synantropijnych / A geographical-historical classification of synanthropic plants. Materiały Zakładu Fitosocjologii Stosowanej UW, 25:32-42. (in Polish)

Kornaś J., 1972. Rozmieszczenie i ekologia rozsiewania się chwastów w zespołach polnych w Gorcach / Distribution and dispersal ecology of weeds in segetal plant communities in the Gorce Mts. (Polish Western Carpathians). Acta Agrobot. 25(1): 5-67. (in Polish)

Kornaś J., 1977. Analiza flor synantropijnych / Analysis of synanthropic floras. Wiad. Bot. 21(2): 85-91. (in Polish)

Kornaś J., 1987. Chwasty polne rozprzestrzeniane z materiałem siewnym. Specjalizacja ekologiczna i procesy wymierania / Speirochoric field weeds: from ecological specialization to extinction. Zesz. Nauk. AR w Krakowie. 216, Sesja Nauk.19: 23-36. (in Polish)

Korniak T., 1992. Flora segetalna północno-wschodniej Polski, jej przestrzenne zróżnicowanie i współczesne przemiany / Segetal flora of north-eastern Poland, its special differentiation and current changes. Acta Acad. Agricult. Tech. Olst. Agricult. 53, Suppl. A: 5-76. (in Polish)

Kubát K., Kaplan Z ., 2001. Arnoseris minima Schweigg. et Koerte in the Czech Republic. Severočes. Přír. Litoměřice, 32: 29-36.

Latowski K., Szmajda P., Żukowski W., 1979. Charakterystyka flory pól uprawnych Wielkopolski na przykładzie wybranych punktów badawczych / Characterization of ploughland flora in Great Poland exemplified by chosen research stations. Bad. Fizjogr. nad Polską Zach. ser. B, 31:65-88. (in Polish)

Mirek Z., Piękoś-Mirkowa H., Zając A. \& Zając M., 2002. Flowering plants and pteridophytes of Poland a checklist. - [In:] Z. Mirek (ed.) Biodiversity of Po- land 1, s. 442. W. Szafer Institute of Botany, Polish Academy of Sciences, Kraków.

Mojski J.E., 1972. Nizina Podlaska [In:] R. Galon (ed.) Geomorfologia Polski T. 2. Niż Polski / Podlasie Lowland [In:] R. Galon (eds). Geomorphology of Poland T. 2. Polish Lowlands. Państwowe Wydawnictwo Naukowe, Warszawa: 318-362. (in Polish)

Nowak A., Nowak S., Spałek K., 2003. Red list of vascular plants of Opole Province. Opole Scient.-Soc. Nature Jurnal, 36: 5-20.

Pinke G., Pal R., Király G., Szendrôdi V., Esterhaázy A ., 2006. The occurrence and habitat conditions of Anthoxanthum puelii Lecoq \& Lamotte and other Atlantic-Mediterranean weed species in Hungary. Journal of Plant Diseases and Protection. Zeitschrift für Pflanzenkrankheiten und Pflanzenschutz. Sonderheft, 22: 587-596.

Prasse R., Ristow M., Klemm G., Machatzi B., Raus T., Scholz H., Stohr G., Sukopp H., Zimmermann F., 2001. Liste der wildwachsenden Gefäßpflanzen des Landes Berlin mit Roter Liste. Hrsg.: Senatsverwaltung für Stadtentwicklung / Der Landesbeauftragte für Naturschutz und Landschaftspflege. Berlin (Kulturbuch-Verlag): 85. (in German).

Ratuszniak I., Sobisz Z., 2006. Flora segetalna Słowińskiego Parku Narodowego / Segetal flora of the Słowiński National Park. Pam Puław. 143: 125-134. (in Polish)

Rutkow sk i L., 2007. Klucz do oznaczania roślin naczyniowych Polski niżowej. Państwowe Wydawnictwo Naukowe, Warszawa: 812. (in Polish)

Sicińs ki J.T., 1998. Ginące i zagrożone gatunki segetalne różnych grup ekologicznych na terenie województwa sieradzkiego / Endangered and threatened segetal species of various ecological groups in the area of the Sieradz Province. Acta Univ. Lodz., Folia bot. 13: 159-164. (in Polish).

Skrzy c z yń ska J., 1994. Studia nad florą i zbiorowiskami Wysoczyzny Siedleckiej / Studies on flora and segetal communities of Siedleckie Upland. Rozprawa habilitacyjna, 39: 1-145. (in Polish)

Skrzyczyńska J., Rzymowska Z ., 2001. Flora segetalna Podlaskiego Przełomu Bugu. / The segetal flora of the Bug river geomorphological territories in the Podlasie region. Acta Agrobot. 54(1): 115-135. (in Polish)

Sowa R., Warcholińska A.U., 1987. Flora synantropijna Łowicza i Skierniewic / Synanthropic flora of Łowicz and Skierniewice. Acta Univ. Lodz. Folia bot. 5: 109-164. (in Polish)

S z o tk ow ski P., 1989. Zmiany we florze i zachwaszczeniu pól południowej części Śląska Opolskiego po 11 latach / Changes in flora and weed infestation on fields in the southern part in Opole Silesia after 11 years. Ibidem: 1-226. (in Polish)

Warcholińska A.U., 1981. Stan i zagrożenie niektórych gatunków chwastów polnych z rodziny Gramineae 
w środkowej Polsce / The status and endangerment of some field weed species from the family Gramineae in Central Poland. Łódz. Tow. Nauk. Sprawozdania z czynności i posiedzeń naukowych 31(11): 1-8. (in Polish)

Wa rcholińska A.U., 1988. Roślinność segetalna terenów rolniczych Puszczy Bolimowskiej i jej współczesne przemiany / Segetal plants of agricultural areas of Bolimów Primeval Forest and its contemporary transformations. Acta Agrobot. 41(2): 369-452. (in Polish)

Warcholińska A.U., 1991. Właściwości i współczesne przemiany flory segetalnej Wzniesień Łódzkich na tle wybranych flor segetalnych środkowej Polski / Features and present changes of the segetal flora of the Eódzkie Upland against the background of some segetal floras of Central Poland. Fragm.. Flor. Geobot. 36(2): 459-497. (in Polish)

Wa rcholińska, A.U., 2002. Właściwości zagrożonych gatunków flory segetalnej środkowej Polski i możliwości jej ochrony / Features of threatened segetal flora species in Central Poland and possibility of their protection. Acta Univ. Lodz. Folia Biol. Oecol. 1: 71-95. (in Polish)

Wójcik Z., 1968. Udział apofitów i antropofitów w zbiorowiskach segetalnych Mazowsza / The participation of apophytes and antropophytes in plant communities of cultivated fields in the Masovia Region. Mat. Zakł. Fitosoc. Stos. UW, 25:109-122. (in Polish)

Urbisz A., Urbisz A., Nowak T.,1998. Występowanie zagrożonych gatunków flory segetalnej na Wyżynie Śląskiej / Occurrence of threatened species of the segetal flora in the Silesian Upland. Acta Univ. Lodz., Folia bot. 13: 225-232. (in Polish)

Zając A., 1979. Pochodzenie archeofitów występujących w Polsce / The orgin of archeophytes occurring in Poland. Uniw. Jagielloński: 1-213. (in Polish)

Zając A., Zając M., Tokarska-Guzik B., 1998. Kenophytes in the flora of Poland: list, status and origin. [In]: J. B. Faliński, W. Adamowski and B. Jackowiak (eds). Synantropization of plant cover in New Polish research. Phytocenosis, 10. Suppl. Cartogr. Geobot. 9: 107-116.

Zając E.U., Zając A., 1975. Lista archeofitów występujących w Polsce / The list of archeophytes occurring in Poland. Zesz. Nauk. UJ, Prace Bot. 395(3): 7-16. (in Polish)
Zając M., Zając A., 1992. A tentative list of segetal and ruderal apophytes in Poland. Zesz. Nauk. UJ, Prace Bot. 24:7-23.

Żukowski W., Jackowiak B. B., 1995: Ginące i zagrożone rośliny naczyniowe Pomorza Zachodniego i Wielkopolski / Endangered and threatened vascular plants of Western Pomerania and Wielkopolska. Zakład Taksonomii Roślin. Univ. A. Mickiewicza w Poznaniu: 1-141 (in Polish).

\section{Flora segetalna Równiny Łukowskiej}

\section{Streszczenie}

Celem pracy była charakterystyka i analiza flory segetalnej Równiny Łukowskiej. Badania prowadzono w latach 2003-2006 na terenie 182 miejscowości. Flora naczyniowa pól uprawnych badanego terenu liczy 305 gatunków należących do 39 rodzin i 168 rodzajów. Na bogactwo gatunkowe ma wpływ zróżnicowanie siedlisk, rozdrobnienie gospodarstw i tradycyjny system gospodarowania.

Analizowana flora cechuje się przewagą gatunków rodzimych $(64,6 \%)$ nad taksonami obcego pochodzenia $(35,4 \%)$. Wśród antropofitów dominują archeofity (27,2\% flory) a $\mathrm{z}$ apofitów najczęściej notowano gatunki ląkowe (23,6\% flory). Gatunki krótkotrwałe mają niewielką przewagę nad wieloletnimi. W obrębie form życiowych dominują terofity $(52,8 \%)$, zwłaszcza wśród antropofitów (85,2\% gatunków tej grupy), ale stosunkowo dużo jest hemikryptofitów $(34,4 \%)$ i geofitów (12,5\%). W analizowanej florze największy udział mają gatunki bardzo rzadkie i rzadkie $(61,6 \%$ flory), natomiast pospolitych i bardzo pospolitych jest $13,1 \%$.

Cechą charakterystyczną jest liczne występowanie gatunków speirochorycznych związanych z ekstensywnym rolnictwem: Agrostemma githago, Bromus secalinus i Avena strigosa. Z gatunków subatlantyckich częstym taksonem jest Arnoseris minima, rzadkim Anthoxanthum aristatum, a Teesdalea nudicaulis występuje bardzo rzadko na badanym terenie. 